\title{
Primary hepatic Ewing sarcoma: a very infrequent case report
}

\author{
Ozturk Ates, ${ }^{1}$ Fatma Bugdaycı Basel, ${ }^{2}$ Nazan Bozdogan, ${ }^{3}$ Bulent Aksel,,${ }^{4}$ Omur Berna Oksuzoglu ${ }^{2}$ \\ ${ }^{1}$ Department of Medical Oncology, Hacettepe University Cancer Institute, Ankara; ${ }^{2}$ Department of Medical Oncology, \\ Dr. Adurrahman Yurtaslan Oncology Training and Research Hospital, Ankara; ${ }^{3}$ Department of Pathology, Dr. Adurrahman \\ Yurtaslan Oncology Training and Research Hospital, Ankara; ${ }^{4}$ Department of General Surgery, Dr. Adurrahman Yurtaslan \\ Oncology Training and Research Hospital, Ankara, Turkey
}

\begin{abstract}
Ewing sarcoma (ES) is a member of small round cell tumors of which contains Wilms' tumor, neuroblastoma, rhabdomyosarcoma and lymphoblastic lymphoma. ES occurs most commonly in the bone but infrequently occurs in the soft tissues without involvement of the bones. Primary involvement of the liver is even rarer. We report the case of 24-year-old female patient with abdominal pain. Abdominal computed tomography scan showed hepatic large cystic lesion. Liver biopsy showed tumoral cells with small narrow stoplasmic and hyper chromatic nucleus evaluated as small round cell tumor. Immunohistochemical study of the lesion revealed CD99 and bcl-2 positive, cytokeratin, LCA, CD138, chromogranin, synaptophysin, myoD1, terminal deoxynucleotidyl transferase and myoglobin negative the diagnosis primary hepatic ES. A research for any other tumor involvement by using positron emission tomography yielded only hepatic involvement revealed. The patient treated with vincristine-cyclophosphamide-adriamycin and ifosfamide-etoposide alternately chemotherapy then hepatic tumor regressed. Patient operated on hepatic wedge resection. She has continued on chemotherapy and she has been doing well at the sixth month of diagnosis.
\end{abstract}

\section{Introduction}

Ewing sarcoma (ES) is a member of small round cell tumors of which contains Wilms' tumor, neuroblastoma, rhabdomyosarcoma and lymphoblastic lymphoma. Ewing's sarcoma family of tumors not only includes ES, but also primitive neuroectodermal tumor

Correspondence: Ozturk Ates, Department of Medical Oncology, Hacettepe University, 06100, Sihhiye, Ankara, Turkey. Tel.: +90.312.305.2929 - Fax: +90.312.305.5000.

E-mail: dr.ozturkates@yahoo.com

Key words: Ewing sarcoma; round cell tumors; primitive neuroectodermal tumor.

Contributions: OA and OBO wrote the paper; NB reviewed the pathological findings; BA operated the patient and FBB assessed the imaging studies. All authors read and approved the final manuscript.

Conflict of interest: the authors declare no potential conflict of interest.

Received for publication: 14 February 2015.

Revision received: 11 June 2015.

Accepted for publication: 15 June 2015.

This work is licensed under a Creative Commons Attribution NonCommercial 4.0 License (CC BY-NC 4.0).

CCopyright O. Ates et al., 2016

Licensee PAGEPress, Italy

Italian Journal of Medicine 2016; 10:67-70

doi:10.4081/itjm.2015.592
(PNET) and Askin's tumor. ES occurs most commonly in the bone but infrequently occurs in the soft tissues without involvement of the bones. Primary ES has been described very rarely in the pancreas, pulmonary, stomach, small bowel, kidney and ovaries. ${ }^{1-3}$ Primary involvement of the liver is even rarer.

In this presented case, primary liver ES in a young female patient who was treated with hepatic wedge resection and chemotherapy.

\section{Case Report}

A 24-year-old Syrian female (refugee) patient was admitted with the complaints of right upper abdominal pain and fatigue. She did not have a history of previous illness or any medication. Physical examination revealed palpable liver at $3 \mathrm{~cm}$ below the costal margin. Abdominal scans revealed $120 \times 100 \mathrm{~mm}$ with cystic mass lesion on the right hepatic lobe (Figure 1). Computed tomography (CT) of the thorax was normal. Pathological examination of liver biopsy revealed as diffuse narrow cytoplasmic and hyper chromatic nucleus small round tumor cells. Immunohistochemical (IHC) study of the lesion showed CD99 and fli-1 (friend leukemia virus integration) positive (Figure 2), cytokeratin (CK), LCA, CD138, chromogranin, synaptophysin, myoD1, terminal deoxynucleotidyl transferase (Tdt), myoglobin and reticulin negative. The morphology of the hepatic lesion and findings of IHC study was consistent with ES. A research for any other tumor involvement by using positron emission tomography yielded only hepatic involvement (SU- 
Vmax: 15). Bone marrow aspiration had not any pathological infiltration. NCI VAC (vincristine $2 \mathrm{mg}$, cyclophosphamide $900 \mathrm{mg} / \mathrm{m}^{2}$, adriamycin $45 \mathrm{mg} / \mathrm{m}^{2}$ first 12 week then $35 \mathrm{mg} / \mathrm{m}^{2}$ ) and IE (ifosfamide 1800 $\mathrm{mg} / \mathrm{m}^{2}$, etoposide $100 \mathrm{mg} / \mathrm{m}^{2}$ ) chemotherapy was started alternately every 3 weeks. At the $9^{\text {th }}$ week, abdominal CT scan revealed a regression in hepatic lesion size $120 \mathrm{~mm}$ to $90 \mathrm{~mm}$. She was operated due to the risk of perforation. Then encapsulated tumor was seen in the exploration. The right liver lobe segments of 4-6 and gallbladder were resected. On cut surface and the hepatic parenchyma was replaced by capsular white mass lesion (Figure 3), which was measured $7.5 \times 6 \times 5 \mathrm{~cm}$. Pathological examination consisted in $95 \%$ of necrosis. She continued on chemotherapy and received $52^{\text {th }} \mathrm{NCI}$ chemotherapy without recurrence. She was doing well at the twelfth month of diagnosis.

\section{Discussion}

ES and PNET are the same group tumors but ES has been used for tumors that lack in the evidence of neuroectodermal differentiation. Both of them are being evaluated within two categories; central and peripheral ES/PNET. Peripheral ES/PNET which occurs in bones and/or extra osseous sites was introduced initially by Arthur Purdy Stout in $1918 .{ }^{4}$ ES has been described in the kidney, parotid gland, chest wall, ovary, rectum or retroperitoneal cavity. The kidney is the most commonly involved visceral organ site however involvement of the liver is very rare. Children and young adults are most commonly affected and the presented patient was under 30 years of age. Patients including ours usually have the complaints of localized pain and swelling. Gross appearance of the ES tumor is multilobulated, soft, friable and rarely cystic formation. It rarely exceeds $10 \mathrm{~cm}$. In the presented case, hepatic cyst formation size is $12 \times 10 \mathrm{~cm}$.

Herein we have presented the case of a patient with primary hepatic Ewing sarcoma. To the best of our knowledge there were four hepatic ES cases in English literature..$^{5-8}$ In our case she had multiloculated cystic liver lesion and diagnosed by CT scan and biopsy. In the English literature this was the second case of primary hepatic ES, which was presented with multiloculated liver cystic lesion. Ozaki et al. reported the first hepatic multiloculated cystic lesion diagnosed with hepatic ES. ${ }^{7}$ In that case a 27 -year-old Japanese woman was admitted with abdominal pain. Multiloculated cystic lesion on liver was revealed with clinical examination. Before patient was operated; mucinous hepatic cyst adenoma had been diagnosed by liver biopsy. But after operation, primary hepatic ES was diagnosed. Differential diagnosis of hepatic cystic lesion comprises simple solitary cystic lesion, poly cystic liver disease and neoplastic lesions such as

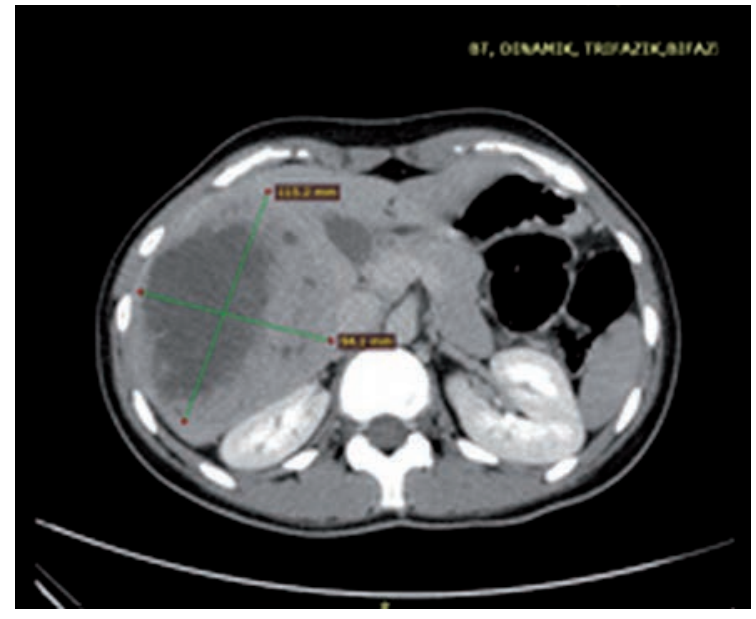

Figure 1. Abdominal computed tomography showed hepatic centrally necrotic cystic lesion.

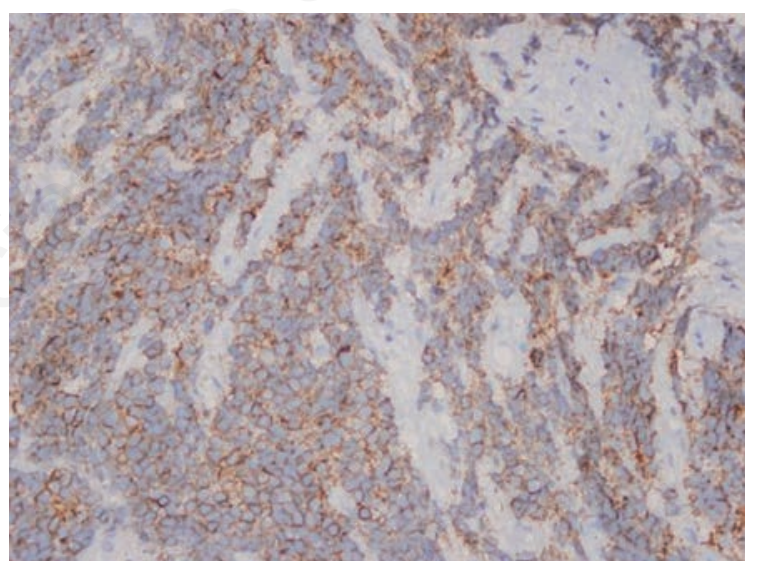

Figure 2. Liver biopsy tumor cells immunoreactivity fli$1(200 x)$.

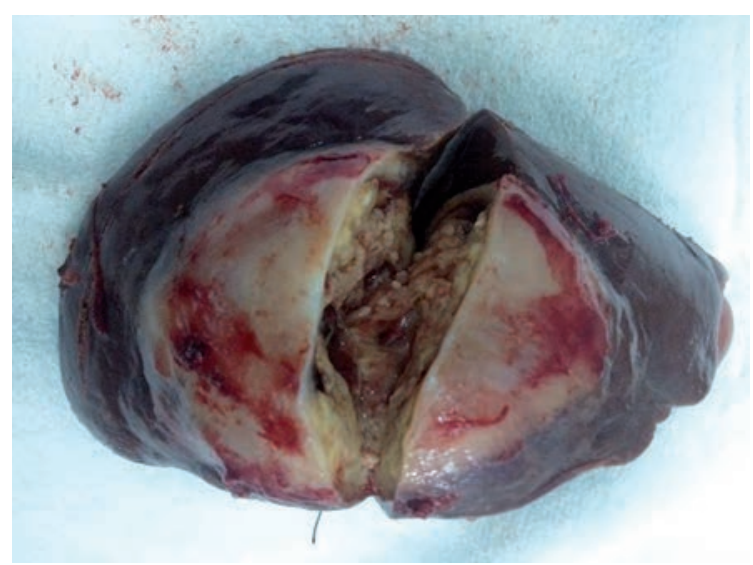

Figure 3. Hepatic wedge resection showing capsuler white mass lesion. 
mucinous cystadenomas, mucinous cystadenocarcinoma, hepatocellular carcinoma and metastasis from other diseases.

There were two cases of hepatic ES in the literature which were presented with hepatic solid mass lesion. ${ }^{6,8}$ The case reported by Cambruzzi et al. was an 18-year-old male showing solid hepatic mass with the complaints of abdominal pain. Preoperative hepatic artery embolization was performed and then the patient was operated for hepatic resection. One month after the surgery, the patient died with pulmonary embolization. ${ }^{6}$

Mani et al. reported a 20-year-old female patient with massive hepatomegaly without mass lesion and bulky renal involvement. The patient was diagnosed with hepatic ES with core biopsy and treated with combined chemotherapy (VAC/IE alternatively). ${ }^{5}$ Over 43 weeks she has been doing well. ${ }^{5}$

Several steps are required for the diagnosis of ES/PNET. ES has specific histological and phenotypic features. The first approach is using light microscopic examination. Histological ES/PNET is composed by monomorphic small blue round cell that reacts positively to glycoprotein p30/32 MIC2 (CD99). The second approach is immunohistochemical consideration. Pathological specimen should be evaluated with immunohistochemical markers including, CK, CD138, a WT1, synaptophysin, muscle specific actin, desmin, myogenin, LCA and leukocyte common antigen (CD45) to exclude other small round cell tumors including neuroblastoma, myeloma, desmoplastic small round cell tumor, mesenchymal chondrosarcoma, alveolar rhabdomyosarcoma, non-Hodgkin's lymphoma and small cell osteosarcoma. ${ }^{8-10}$ Neuroblastoma shows immunoreactivity to NSE, S-100 and Leu-7 like ES, but negative for vimentin. Lymphoblastic lymphoma is immunoreactive to CD99 but different in that it tests positive to CD45 and Tdt. Rhabdomyosarcoma is immunoreactive to myogenin, myo-D1, desmin and actin differs from Ewing sarcoma. In our patient, IHC showed CD99 and bcl-2 positive, CK, LCA, CD138, chromogranin, synaptophysin, myoD1, Tdt and myoglobin negative.

The translocation $\mathrm{t}(11,22)$ (q24; q12) is specific of Ewing sarcoma and resulting in the production of the $E W S / F l i-1$ fusion gene. This fusion gene expresses CD 99 antigen (MIC 2) and occurs in $85 \%$ of patients with ES/PNET. The remaining of patients have variant of this translocation. ${ }^{11,12}$ in our case liver biopsy is strongly positive for CD99 but we were unable to work the $\mathrm{t}(11: 22)$ (q24:q12) translocation due to ours genetic laboratory having no special based translocation assay kits.

Treatment of ES needs multidisciplinary approach. A high initial complete response of $94 \%$ was observed in patients of PNET treated with VAC chemotherapy plus local radiation therapy. ${ }^{13}$ The best response was reported with combinations based on anthracyclines (doxorubicin) and high doses of alkylation agents (cyclophosphamide or ifosfamide). ${ }^{14,15}$ Despite high responses with multimodal therapy, most patients have a rapid progression. For the presented case, VAC/IE chemotherapy alternatively was started. After 9 cycles of chemotherapy tumor was regressed and the patient was operated. After 52 weeks of treatment, the patient was in good condition.

The prognosis of patients with hepatic ES is very poor especially in the metastatic stage and standard therapy approach is still unclear. But multimodal therapy may improve clinical symptoms. Prognostic factors of ES are the presence of metastatic disease at the time of initial diagnosis, large tumor size, extensive necrosis and poor response of initial chemotherapy.

\section{Conclusions}

As conclusion clinicians should carefully evaluate young patients who have cystic and necrotic hepatic lesions to exclude hepatic ES.

\section{References}

1. Pomara G, Cappello F, Cuttano MG, et al. Primitive neuroectodermal tumor (PNET) of the kidney: a case report. BMC Cancer 2004;4:3.

2. Colovic RB, Grubor NM, Micev MT, et al. Perigastric extraskeletal Ewing's sarcoma: a case report. World J Gastroenterol 2009;15:245-7.

3. Welsch T, Mechtersheimer G, Aulmann S, et al. Huge primitive neuroectodermal tumor of the pancreas: report of a case and review of the literature. World J Gastroenterol 2006;12:6070-3.

4. Stout AP. A tumor of the ulnar nerve. Proc N Y Pathol Soc 1918;12:2-12

5. Mani S, Dutta D, De BK. Rare primitive neuroectodermal tumor (PNET) of liver in a young woman. Gastrointest Cancer Res 2011;4:111-3

6. Cambruzzi E, Guerra EE, Hilgert HC, et al. Primitive neuroectodermal tumor of the liver: a case report. Case Rep Med 2011;2011:748194.

7. Ozaki Y, Miura Y, Koganemaru S, et al. Ewing sarcoma of the liver with multilocular cystic mass formation: a case report. BMC Cancer 2015;15:16.

8. Huang SF, Chiang JH, Jan HC, et al. Intra-abdomen Ewing's sarcoma. ANZ J Surg 2011;81:377.

9. Vakar-López F, Ayala AG, Raymond AK, Czerniak B. Epithelial phenotype in Ewing's sarcoma/primitive neuroectodermal tumor. Int J Surg Pathol 2000;8:59-65.

10. Kushner BH, Hajdu SI, Gulati SC, et al. Extracranial primitive neuroectodermal tumors: the memorial sloankettering cancer center experience. Cancer 1991;67: 1825-29.

11. Le Deley MC, Delattre O, Schaefer KL, et al. Impact of 
EWS-ETS fusion type on disease progression in Ewing's sarcoma/peripheral primitive neuroectodermal tumor: prospective results from the cooperative EuroE.W.I.N.G. 99 trial. J Clin Oncol 2010;28:1982-8.

12. Zucman J, Delattre O, Desmaze C, et al. Cloning and characterization of the Ewing's sarcoma and peripheral neuroepithelioma $t(11 ; 22)$ translocation breakpoints. Genes Chromosomes Cancer 1992;5:271-7.

13. De Alava E, Gerald WL. Molecular biology of the
Ewing's sarcoma/primitive neuroectodermal tumor family. J Clin Oncol 2000;18:204-13.

14. Jürgens H, Bier V, Harms D, et al. Malignant peripheral neuroectodermal tumors: a retrospective analysis of 42 patients. Cancer 1988;61:349-57.

15. Smorenburg CH, van Groeningen CJ, Meijer OW, et al. Ewing's sarcoma and primitive neuroectodermal tumour in adults: single-centre experience in The Netherlands. Neth J Med 2007;65:132-6. 